\title{
A NUMERICAL SCHEME FOR THE ELECTROMAGNETIC RESPONSE IN THIN CONDUCTORS OF ARBITRARY PLANAR SHAPE
}

\author{
P. F. SIEW ${ }^{1}$ \\ (Received 14 July 1992; revised 5 October 1992)
}

\begin{abstract}
A calculation of the electromagnetic response of a thin conductor in the presence of an exciting primary magnetic field has been attempted by various authors. Analytic solutions are obtainable when either the conductor is of infinite extent or when the problem possesses some symmetry. The loss of symmetry makes the problem difficult to solve except for the simplest shape - that of a circular conductor. A numerical method has been used for the rectangular conductor by other authors. In this paper we consider the response due to a thin plane conductor of arbitrary shape. The method involves the numerical generation of a set of body-fitted orthogonal curvilinear coordinates which maps the conductor onto a unit square. Good orthogonal grids can be generated for shapes that do not deviate too far from the rectangular. In terms of these curvilinear coordinates the vector potential for the area current density satisfies an integro-differential equation which is solved numerically.
\end{abstract}

\section{Introduction}

Problems involving the induction of eddy currents in the low-frequency limit, are important in several branches of physics, including mining geophysics. In the electromagnetic method of prospecting, an oscillating magnetic field is set up by sending an alternating current through a transmitting loop of wire and the presence of a conductor is detected by the presence of an anomalous secondary field due to the eddy currents induced in the conductor. The electromagnetic response of idealized models whose thickness is small compared to its lateral

\footnotetext{
${ }^{1}$ School of Maths and Stats, Curtin University of Technology, Perth, Western Australia 6001. (C) Australian Mathematical Society, 1994, Serial-fee code 0334-2700/94
} 
extent is of interest in the detection of ores occuring in lateritic formation. It is also of interest in ionospheric physics and in the induction of oceanic basins.

The induction of eddy currents in thin sheets has been studied by various authors. The method used usually depends on the geometry of the conductor. The simplest case is when the conductor is considered to be an infinite thin sheet [7], or when certain assumptions are made regarding the inducing field $[1,9]$. When the conductor is of finite extent, or when there is no axial symmetry, the problem is not always solvable analytically. Siew and Hurley [10] have obtained analytical solutions for the response in thin circular conductors in the presence of an arbitrary exciting field. The analytical methods used are unfortunately not suitable for other geometries. Lamontagne and West [6] used a numerical formulation for the rectangular conductor, but here the scheme is geometry dependent. There is therefore a need for a general scheme that will not depend on the geometry of the conductor and it is the purpose of this paper to outline one such scheme.

\section{Basic equations}

The formulation follows quite closely that used in Siew and Hurley [10]. We consider here a plane conductor with normal in the $O z$ direction. The conductor is assumed to be surrounded by vacuum in which there is a primary alternating magnetic field $\mathbf{H}^{(p)} \exp (i \omega t)$, and the eddy currents induced in the conductor give rise to an anomalous field $\mathbf{H}^{(s)} \exp (i \omega t)$. The time variation of $\exp (i \omega t)$ will be assumed from now on. Outside the conductor, Maxwell's equation for the electric field $\mathbf{E}$ is given by

$$
\nabla \times \mathbf{E}=-i \omega \mu_{0}\left(\mathbf{H}^{(p)}+\mathbf{H}^{(s)}\right),
$$

where $\mu_{0}$ is the permeability of free space. If the thickness $d$ of the conductor is small enough so that the current density $\mathbf{J}$ may be assumed constant across it, an area current density $\mathbf{K}$ may be defined [6] as

$$
\mathbf{K}=\int_{0}^{d} \mathbf{J} d z=\mathbf{J} d .
$$

Then, on the surface of the conductor, (2.1) may be replaced by

$$
(\nabla \times \mathbf{K}) \cdot \hat{\mathbf{z}}=-i \omega \sigma \mu_{0} d\left(H_{z+}^{(p)}+H_{z+}^{(s)}\right),
$$


where $\hat{\mathbf{z}}$ is the unit vector in the $O z$ direction, $\sigma$ is the conductivity, assumed constant, and the subscripts $z+$ denote the vertical components of the magnetic fields. The right hand side of (2.2) is obtained by assuming the fields do not vary appreciably across the thickness of the conductor. $\mathbf{K}$ may also be defined in terms of a stream potential $U(x, y)$ by the relation (see for example, Smythe [11]),

$$
\mathbf{K}=\nabla \times(0,0, U)
$$

and the equation to be solved is given in non-dimensional form by

$$
\nabla^{2} U=i \alpha\left\{H_{z_{+}}^{(p)}+H_{z_{+}}^{(s)}\right\}
$$

where $\alpha=\mu_{0} \sigma \omega d a$ is the induction number, and $a$ is a suitable length scale.

Equation (2.3) is solved with the condition that $U=0$ on the boundary of $S$. This was done analytically for the circular conductor by Siew and Hurley [10] and numerically by Lamontagne and West [6] for a rectangular conductor. In terms of the integral form of the Biot Savart law we have

$$
H_{z}^{(s)}(x, y, 0+)=H_{z_{+}}^{(s)}=\frac{1}{4 \pi} \iint_{S} \frac{\left(\mathbf{r}-\mathbf{r}^{\prime}\right) \cdot \nabla U\left(\mathbf{r}^{\prime}\right)}{\left|\mathbf{r}-\mathbf{r}^{\prime}\right|^{3}} d^{2} \mathbf{r}^{\prime},
$$

where $S$ is the surface of the conductor, $\mathbf{r}$ and $\mathbf{r}^{\prime}$ are the observation and source points respectively, and we have implicitly assumed that $\left\{\left(\mathbf{r}-\mathbf{r}^{\prime}\right) /\left|\mathbf{r}-\mathbf{r}^{\prime}\right|^{3}\right\}$ does not vary appreciably across the thickness of the conductor. As remarked in [6], the present model is appropriate for a large proportion of geologically occuring conductors. Equation (2.4) is well defined except where $\mathbf{r}^{\prime}=\mathbf{r}$ when it becomes an improper integral. When $\mathbf{r}$ lies inside $S$ it can be shown that the improper integral is convergent in the usual sense while, if $\mathbf{r}$ is a boundary point of $S$, the integral becomes logarithmic, an edge property that was also encountered in Hurley and Siew [4]. Here the logarithmic singularity is a consequence of the averaging process used to derive (2.4). This last equation holds everywhere except for a region that is $O(d)$ from the boundary of the thin conductor. Given that we are solving for $U$ at interior points on the surface, the logarithmic singularity is never a feature of the problem.

\section{A numerical scheme for a unit square}

It is useful to consider initially a conductor occupying the unit square $0 \leq$ $x \leq 1$ and $0 \leq y \leq 1$. For other geometries the conductor can be mapped onto 
a unit square leading to an algebraically more complicated integro-differential equation, but little detracts from the method described in this section. In both cases, the right hand side of (2.4) still presents a problem numerically. In the numerical scheme for a rectangular conductor, Lamontagne and West used a difference approximation for the derivatives of $U$ and imposed a quadrature approximation on $\nabla U$ which removes the singularity. However the method becomes unwieldy when the shape of the conductor is not rectangular and when it is necessary to map its boundary onto that of a unit square. Now, the convergence of the improper integral defining $H_{z_{+}}^{(s)}$ means we can omit a small neighbourhood of $\mathbf{r}^{\prime}=\mathbf{r}$ when evaluating the surface integral. Analytically the integral over this small neighbourhood can be shown to tend to zero as its area tends to zero. Therefore, in the numerical scheme adopted below, we omit the contribution from the four sub-regions surrounding the observation point. A numerical justification for this is given in the appendix where we show that a different quadrature can be chosen for $U$ over these four squares such that the contribution is negligible or zero.

Consider now a regular $N \times N$ mesh covering the unit square. It is required to obtain estimates for $U_{m n}=U\left(x_{m}, y_{n}\right)$ at the observation point $\left(x_{m}, y_{n}\right)$ satisfying (2.3) where $x_{m}=m h$ and $y_{n}=n h$ for $1 \leq m \leq N-1,1 \leq n \leq N-1 . U_{m n}=0$ for $m$ and $n$ equal to 0 or $N$, being points on the boundary of the conductor. If suffixes $i$ and $j$ are used to replace $m$ and $n$ respectively for the source points in the integrand in (2.4), and if the integral over the square $\Delta_{i j}$ occupying the region $x_{i} \leq x \leq x_{i+1}$ and $y_{j} \leq y \leq y_{j+1}$ is denoted by $I_{i j m n}$ then (2.3) and (2.4) yield

$$
\left(\nabla^{2} U\right)_{m n}=i \alpha\left\{P_{m n}+\sum_{i, j} I_{i j m n}-I_{4}\right\},
$$

where $\left(\nabla^{2} U\right)_{m n}=U_{m+1, n}+U_{m-1, n}-4 U_{m n}+U_{m, n-1}+U_{m, n+1}$ is the Laplacian, $P_{m n}$ is the value of $H_{z+}^{(p)}\left(x_{m}, y_{n}\right)$ and $I_{4}$ denotes the contribution from the four squares surrounding the observation point, that is, for $i=m-1$ or $m$ and $j=n-1$ or $\mathrm{n}$. In $\Delta_{i j}$, we first interpolate $U(x, y)$ by using the values at the four comers of the square:

$$
U\left(x^{\prime}, y^{\prime}\right)=l_{i j} U_{i+1, j+1}-l_{i, j+1} U_{i+1, j}+l_{i+1, j+1} U_{i, j}-l_{i+1, j} U_{i, j+1} \text {, }
$$

where $\left(x^{\prime}, y^{\prime}\right) \in \Delta_{i j}$ and

$$
l_{i j}=\left(x^{\prime}-i h\right)\left(y^{\prime}-j h\right) / h^{2}
$$


Equation (3.2) also yields

$$
\frac{\partial U}{\partial x^{\prime}}=\frac{1}{h^{2}}\left\{\left[y^{\prime}-(j+1) h\right]\left[U_{i j}-U_{i+1, j}\right]+\left[y^{\prime}-j h\right]\left[U_{i+1, j+1}-U_{i, j+1}\right]\right\}
$$

and

$$
\frac{\partial U}{\partial y^{\prime}}=\frac{1}{h^{2}}\left\{\left[x^{\prime}-i h\right]\left[U_{i+1, j+1}-U_{i+1, j}\right]+\left[x^{\prime}-(i+1) h\right]\left[U_{i j}-U_{i, j+1}\right]\right\} .
$$

Thus, $I_{i j m n}$ takes the form

$$
\int_{j h}^{(\jmath+1) h} \int_{i h}^{(i+1) h} \frac{\mathcal{G}_{i j m n}\left(x^{\prime}, y^{\prime}\right)}{h^{2}\left[\left(n h-y^{\prime}\right)^{2}+\left(m h-x^{\prime}\right)^{2}\right]^{3 / 2}} d x^{\prime} d y^{\prime},
$$

where

$$
\mathcal{G}_{i j m n}\left(x^{\prime}, y^{\prime}\right)=U_{i j} g_{i+1, j+1, m, n}-U_{i, j+1} g_{i+1, j, m, n}-U_{i+1, j} g_{i, j+1, m, n}+U_{i+1, j+1} g_{i, j, m, n}
$$

and

$$
g_{i, j, m, n}=\left(m h-x^{\prime}\right)\left(y^{\prime}-j h\right)+\left(n h-y^{\prime}\right)\left(x^{\prime}-i h\right) .
$$

For each value of $i$ and $j, \Delta_{i j}$ is mapped onto the square $-1 \leq \bar{x} \leq 1$, $-1 \leq \bar{y} \leq 1$ by use of the transformation

$$
x=h[\bar{x}+2 i+1] / 2, \quad y=h[\bar{y}+2 j+1] / 2
$$

and the resulting integral evaluated by use of a Gauss-Legendre quadrature. In the various examples shown in this paper we have used an 8-point GaussLegendre quadrature (see Stroud and Secrest [12]) - using a higher order quadrature does not give significantly different results. Once all the coefficients of $U_{m n}$ are found, the resulting $(N-1)^{2} \times(N-1)^{2}$ complex system of equations given by $(3.1)$ is solved by LU-factorization. As shown in the appendix, $I_{4}$ is identically zero for a suitable quadrature approximation for $U$ slightly different from (3.2) above.

\section{Body fitted coordinates}

When the cross-section of the conductor is not square, the choice of the numerical technique becomes of importance. There are many contenders here, and all schemes have some inherent deficiencies either in terms of computational 
complexities or smoothness of the estimated quantities. Directly connected with this is the coverage of the actual region in question. As pointed out by Formberg [3] conformal mapping is an ill-conditioned problem since small changes in the domain may result in large displacements of neighbouring points in the image. This is not a dismissal of the technique which has many useful applications; indeed, in a recent paper, Kang and Leal [5] used a conformal map as a preliminary step for their boundary integral technique. This technique may be used for more complicated shapes than the ones treated in this paper. Finite elements have been extensively used when dealing with bodies with curved boundaries, however, not all derivatives can be made continuous across the boundaries of the various elements. The use of body fitted coordinates has made finite difference schemes a worthwhile alternative.

In this paper we use a numerical scheme to obtain a set of body fitted coordinates which maps the boundary of the conductor onto a unit square. The method used to obtain the orthogonal grid is that derived by Ryskin and Leal [8]. The choice is influenced by the desire to retain some control over the boundary correspondence between the original domain and its image and still work with an orthogonal set of coordinates. For this particular application, orthogonality leads to a comparatively simpler transformed equation. Another advantage is that the arbitrary domain in the $O x y$ frame of reference is always mapped onto the unit square $0 \leq \xi \leq 1,0 \leq \eta \leq 1$ in the $O \xi \eta$ frame, over which a regular $N \times N$ mesh is imposed. We give a brief description of the method below and the interested reader is referred to the original paper [8] for details. Since $x$ and $y$ are the cartesian coordinates which are linear scalar functions of position, $\operatorname{grad}(x)$ and $\operatorname{grad}(y)$ are constant valued vector fields and hence

$$
\nabla^{2} x=0, \quad \nabla^{2} y=0
$$

everywhere, where $\nabla^{2}$ is the covariant Laplace operator. Equation (4.1) is independent of the coordinates chosen and hence may be used as the defining equations for the functions

$$
x=x(\xi, \eta), \quad y=y(\xi, \eta)
$$

which map the cartesian coordinates to the set of curvilinear coordinates $(\xi, \eta)$. If the scale factors along curves of constant $\eta$ and $\xi$ are respectively $h_{1}$ and $h_{2}$, then (4.1) becomes

$$
\frac{\partial}{\partial \xi}\left(f \frac{\partial x}{\partial \xi}\right)+\frac{\partial}{\partial \eta}\left(\frac{1}{f} \frac{\partial x}{\partial \eta}\right)=0, \quad \frac{\partial}{\partial \xi}\left(f \frac{\partial y}{\partial \xi}\right)+\frac{\partial}{\partial \eta}\left(\frac{1}{f} \frac{\partial y}{\partial \eta}\right)=0,
$$


where $f(\xi, \eta)=h_{2} / h_{1}$ is a distortion function in the sense that it measures how far the mapping deviates from being conformal (this being the case when $f=1$ ). Now, in general there are two degrees of freedom in choosing the mapping function. The requirement for orthogonality (see (4.5) below) means that we can only impose one other constraint. One choice is to specify the distribution for $f(\xi, \eta)$ in the domain in question. This choice does not allow us to impose any control over the boundary correspondence. An alternative choice is to prescribe the boundary correspondence while allowing the values of $f(\xi, \eta)$ to be determined by the mapping itself. This is what the authors referred to as the weak constraint method. Chikhliwala and Yortsos [2] have shown that the method of weak constraint gives satisfactory orthogonal grid when (4.3) is solved with a mix of Dirichlet and Neumann conditions on the boundary. In practice, one starts with an initial guess for $x(\xi, \eta), y(\xi, \eta)$ from which the values of $f(\xi, \eta)$ on the boundary can be deduced. The boundary values of $f(\xi, \eta)$ are then used to obtain a smooth interpolation of its distribution inside the unit square via the equation

$$
f(\xi, \eta)=(1-\xi) f(0, \eta)+\xi f(1, \eta)+(1-\eta) f(\xi, 0)+\eta f(\xi, 1)-f^{c}(\xi, \eta),
$$

where

$$
f^{c}(\xi, \eta)=(1-\xi)(1-\eta) f(0,0)+(1-\xi) \eta f(0,1)+\xi(1-\eta) f(1,0)+\xi \eta f(1,1) .
$$

Equation (4.3) is then solved with the prescribed boundary conditions and the whole process repeated until some convergence criteria is satisfied.

The actual geometry of the region under study determines the ease with which the grids are obtained. Since the problem is two dimensional, a condition for orthogonality is given by requiring that

$$
\frac{\partial x}{\partial \xi} \frac{\partial x}{\partial \eta}+\frac{\partial y}{\partial \xi} \frac{\partial y}{\partial \eta}=0 .
$$

This equation further implies that

$$
f \frac{\partial x}{\partial \xi}=\frac{\partial y}{\partial \eta}, \quad \frac{\partial x}{\partial \eta}=-f \frac{\partial y}{\partial \xi} .
$$

Numerically the value of

$$
M=\cos ^{-1}\left\{\frac{1}{h_{1} h_{2}}\left[\frac{\partial x}{\partial \xi} \frac{\partial x}{\partial \eta}+\frac{\partial y}{\partial \xi} \frac{\partial y}{\partial \eta}\right]\right\}
$$


gives a measure of the orthogonality achieved. Similar to Chikhliwala and Yortsas we adopt as a measure of orthogonality the quantity $M D O=(0.5-$ $M / \pi)$. Good orthogonal grids are obtained when $M D O$ is small. This value is calculated at each grid point and the maximum value is used as one of the criteria for terminating the iterations. The other criterion is the maximum absolute difference $\Delta f$ between successive values of the distortion function. In the examples considered here $\Delta f=0.01$ has been used in each case.

For any given geometry, (4.3) is replaced by the scheme

$$
a_{1} z_{i+1}+a_{2} z_{l-1}+a_{3} z+a_{4} z_{j-1}+a_{5} z_{j+1}=0,
$$

where $z$ is either $x$ or $y$, suffixes $i$ and $j$ are suppressed (that is $z_{i+1, j}$ is written as $z_{t+1}$ only) and $a_{k}, k=1,2, \ldots, 5$ are coefficients involving $f$. Equation (4.7) is solved using an SOR scheme with prescribed Dirichlet or Neumann conditions on the boundary of the region. The number of iterations required for convergence is small if one starts with a low number of meshes. To obtain good orthogonal grids with $N=20$ say, one might start with an arbitrary distribution of values for $\left(x\left(\xi_{i}, \eta_{j}\right), y\left(\xi_{i}, \eta_{j}\right)\right)$ over a $5 \times 5$ mesh. A higher convergence tolerance may be used at this stage. The set of grid values obtained is then linearly interpolated over a $10 \times 10$ mesh and the iterations carried out with a lower convergence tolerance. The set of values so obtained is then interpolated over the final $20 \times 20$ mesh and the iterations again carried out this time with the prescribed convergence criteria. The program exits either when the minimum value of $M D O$ is achieved or when the deviation in the distortion function is everywhere less than the value set for $\Delta f$. The final set of points $\left(x\left(\xi_{i}, \eta_{j}\right), y\left(\xi_{i}, \eta_{j}\right)\right)$ are then used in solving the equivalent transformed equation obtained from (2.3).

\section{The general case}

Starting with (2.3), the transformation given by (4.2) leads to the equation

$$
\begin{gathered}
\frac{1}{J}\left\{f \frac{\partial^{2} U}{\partial \xi^{2}}+\frac{1}{f} \frac{\partial^{2} U}{\partial \eta^{2}}-\frac{1}{J} \frac{\partial U}{\partial \xi}\left[\frac{\partial x}{\partial \xi}\left(f^{2} \frac{\partial^{2} x}{\partial \xi^{2}}+\frac{\partial^{2} x}{\partial \eta^{2}}\right)+\frac{\partial y}{\partial \xi}\left(f^{2} \frac{\partial^{2} y}{\partial \xi^{2}}+\frac{\partial^{2} y}{\partial \eta^{2}}\right)\right]\right. \\
\left.-\frac{1}{J} \frac{\partial U}{\partial \eta}\left[\frac{\partial x}{\partial \eta}\left(\frac{\partial^{2} x}{\partial \xi^{2}}+\frac{1}{f^{2}} \frac{\partial^{2} x}{\partial \eta^{2}}\right)+\frac{\partial y}{\partial \eta}\left(\frac{\partial^{2} y}{\partial \xi^{2}}+\frac{1}{f^{2}} \frac{\partial^{2} y}{\partial \eta^{2}}\right)\right]\right\} \\
=i \alpha H_{z_{+}}^{(p)}(x(\xi, \eta), y(\xi, \eta))+\frac{i \alpha}{4 \pi} \iint_{\Omega} \frac{G\left(\xi, \eta ; \xi^{\prime}, \eta^{\prime}\right)}{\left[\left(x-x^{\prime}\right)^{2}+\left(y-y^{\prime}\right)^{2}\right]^{3 / 2}} d \xi^{\prime} d \eta^{\prime},(5.1 \mathrm{a})
\end{gathered}
$$


where

$$
\begin{aligned}
G\left(\xi, \eta ; \xi^{\prime}, \eta^{\prime}\right)= & {\left[\left(x-x^{\prime}\right) \frac{\partial y^{\prime}}{\partial \eta^{\prime}}-\left(y-y^{\prime}\right) \frac{\partial x^{\prime}}{\partial \eta^{\prime}}\right] \frac{\partial U}{\partial \xi^{\prime}} } \\
- & {\left[\left(x-x^{\prime}\right) \frac{\partial y^{\prime}}{\partial \xi^{\prime}}-\left(y-y^{\prime}\right) \frac{\partial x^{\prime}}{\partial \xi^{\prime}}\right] \frac{\partial U}{\partial \eta^{\prime}} }
\end{aligned}
$$

$J$ is the Jacobian given by

$$
J=\frac{\partial x}{\partial \xi} \frac{\partial y}{\partial \eta}-\frac{\partial x}{\partial \eta} \frac{\partial y}{\partial \xi}
$$

$\Omega$ denotes the unit square in the $O \xi \eta$ frame and use has been made of (4.5) and (4.6) to give the relation $J=f h_{1}^{2}$. Also, it is understood that $x$ and $y$ are functions of $\xi$ and $\eta$ while $x^{\prime}$ and $y^{\prime}$ are functions of $\xi^{\prime}$ and $\eta^{\prime}$. We note in passing that when the conductor is rectangular, then $x, y$ are linear in $\xi, \eta$ respectively and we recover (2.3) and (2.4).

We assume now that the orthogonal grids have been generated by the method of Section 4. The left hand side of (5.1a) is discretized using central differences and the discretized form of the right hand side looks similar to the right hand side of (3.1), but with a slightly more complicated expression for $I_{l \jmath m n}$ and $I_{4}$.

\section{Some numerical results}

We present here some results obtained using the numerically generated grids to solve (5.1). Figure 1 shows the stream potential maps for a $2 \times 3$ rectangular thin plate due to a uniform vertical inducing field with $\alpha=20$. The plate occupies the region $0 \leq x \leq 3,0 \leq y \leq 2$. This region is mapped onto a unit square which is superimposed with a $20 \times 20$ grid and the resulting $361 \times 361$ system of equations are then solved for $U$. Figure 2 gives the vertical component of the induced magnetic field along a traverse starting from the point with coordinates $(-2,1,0.2)$ to the point $(5,1,0.2)$.

Figures 3 and 4 give the corresponding results for the response due to a unit vertical dipole positioned at $(1.5,4,0.2)$. The traverse path used in Figure 4 is from the point with coordinates $(1.5,-2,0.2)$ to the point $(1.5,4,0.2)$.

Good orthogonal grids may be obtained for shapes that do not deviate too much from the rectangular. Figure 5 shows the $40 \times 40$ grid that is generated for the quadrilateral shown. The average deviation from orthogonality is 0.52 of a degree and is worse near the obtuse angled top right hand comer where it 


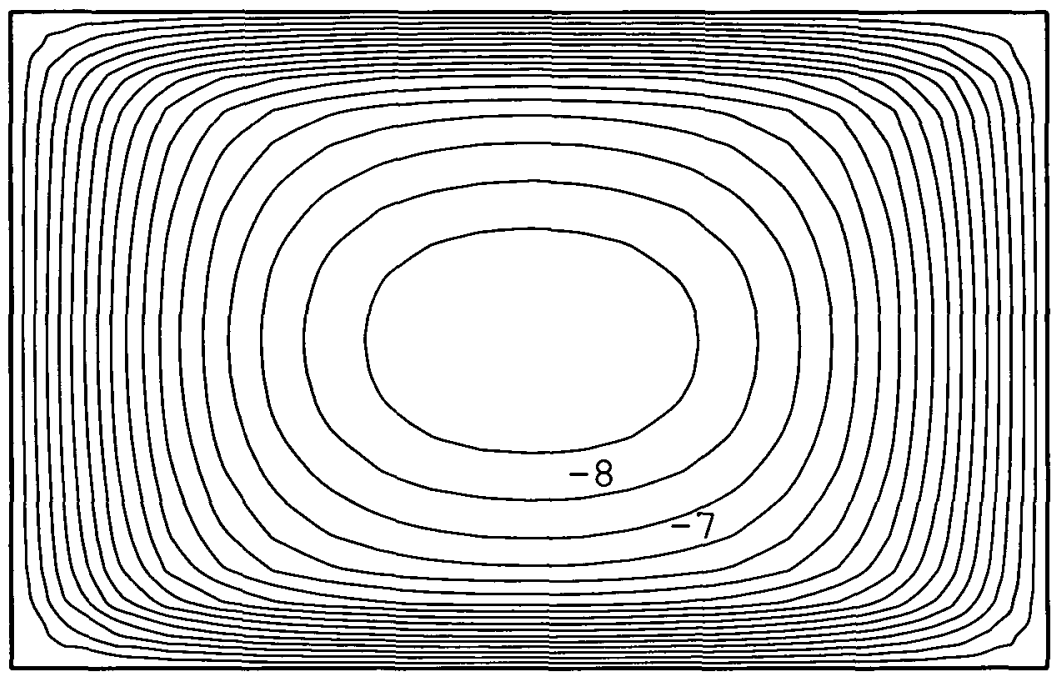

(a)

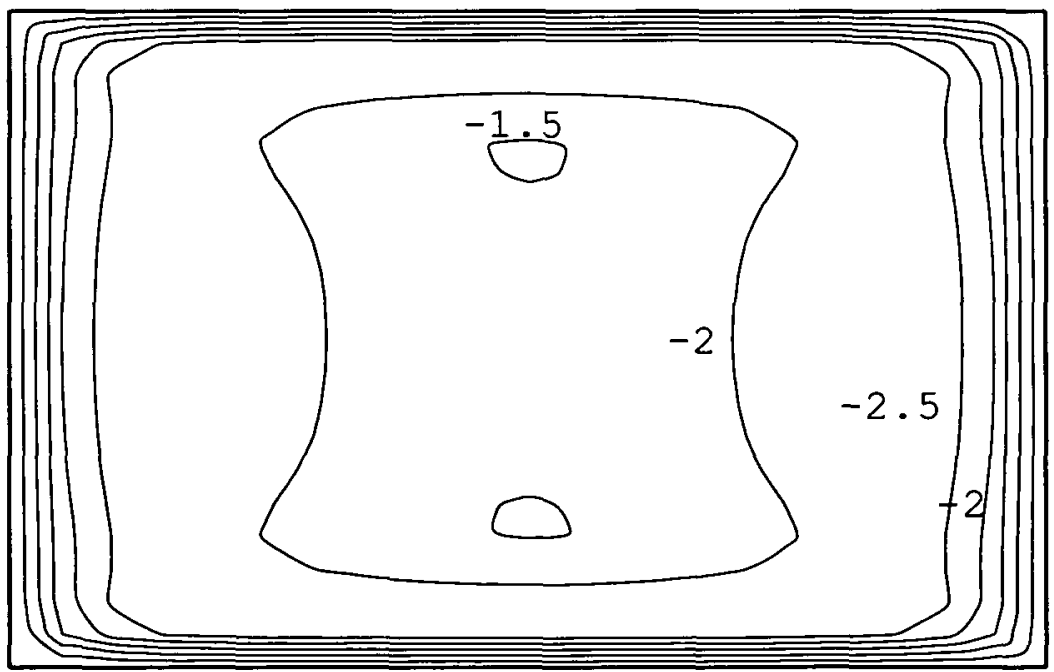

(b)

FIGURE 1. The stream potential maps due to a uniform vertical primary field. (a) In-phase component. (b) Quadrature component. $\alpha=20$. Zero contour on boundaries. Contour intervals $=0.5$. (Dimension of plate not to scale) 


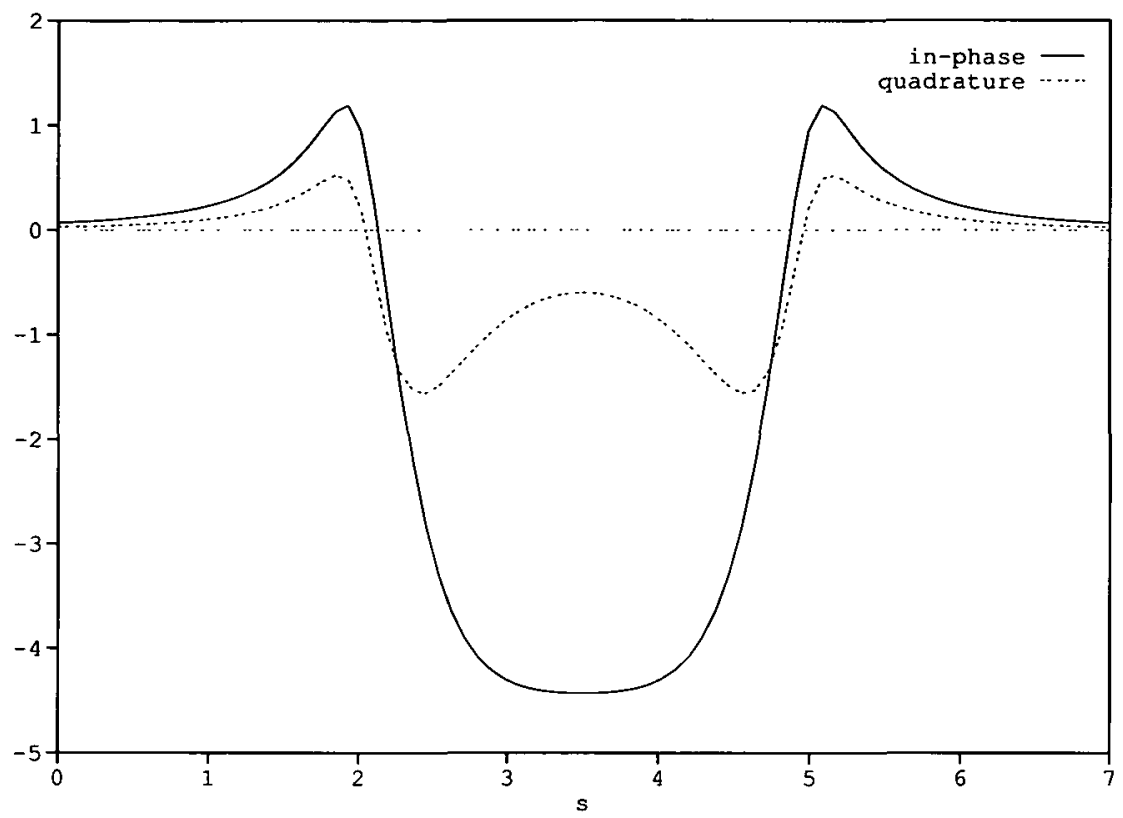

FIGURE 2. The vertical component of the induced magnetic field due to a uniform primary field. $\alpha=20$ The horizontal scale $s$, measures distances along the path of traverse from $(-2,1,0.2)$ to $(5,1,0.2)$. The response over the plate covers the range $2 \leq s \leq 5$.

deviates by a little over five degrees. Figure 6 shows the corresponding $40 \times 40$ grid that is generated for the shape where the right most boundary curve has equation $x=2+0.5 \cos (\pi y / 2)$. In this case all corners are right angles and the generated grids have an average deviation from orthogonality of about half a degree, the worse being near the bottom right hand corner where it reached 0.89 of a degree. This may be improved on by setting lower convergence criteria on $M D O$ and $\triangle f$.

In both Figures 5 and 6, Dirichlet conditions are imposed on the right hand boundary while zero Neumann conditions are maintained on the other boundaries. Figure 7 illustrates the change in the grids generated when the distortion function is changed. In both cases, Dirichlet conditions are maintained only on the curved boundary. For this slightly awkward shape, the entire boundary $\eta=1$ in the tranformed plane is mapped onto a point in the physical plane. In Figure 8 we show the stream potential maps for the shape of Figure 6 with a unit vertical dipole situated at $(3,1,0.2)$. The corresponding induced magnetic field as calculated along a horizontal path given by $y=1$ and at the same height as the inducing dipole is shown in Figure 9. 


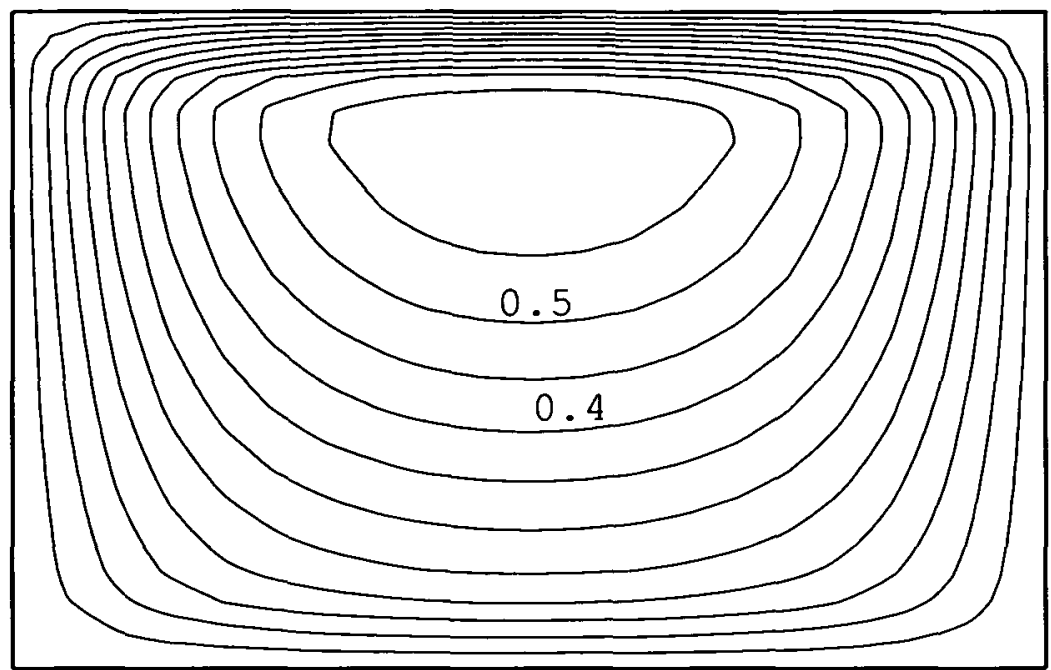

(a)

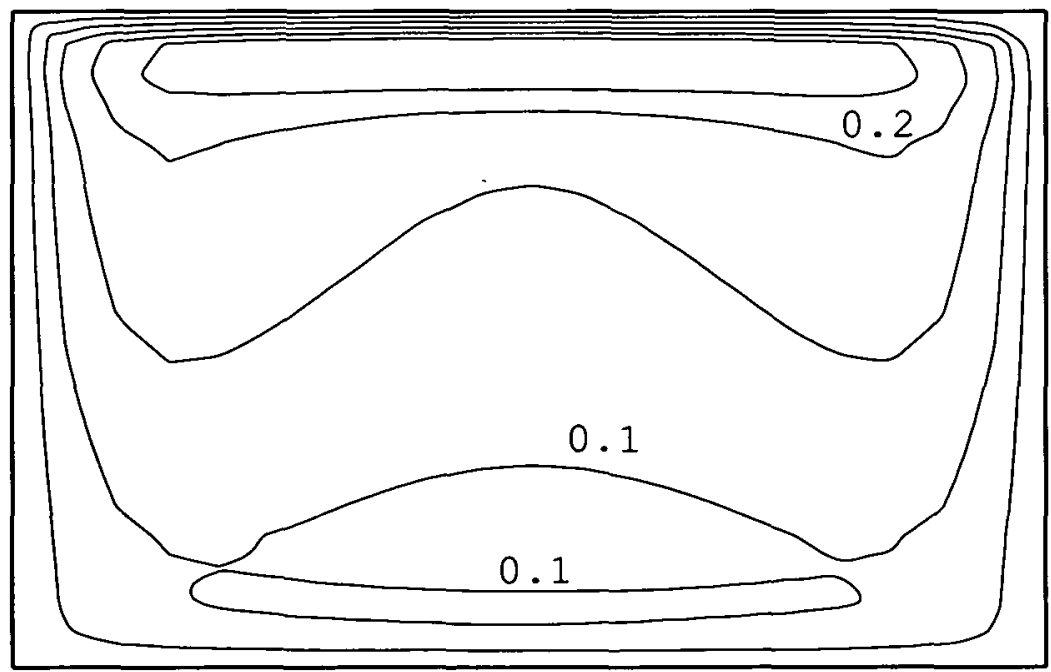

(b)

FIGURE 3. The stream potential maps for the rectangular plate with a primary unit vertical dipole situated at $(1.5,4,0.2)$. (a) In-phase component. (b) Quadrature component. $\alpha=20$. Zero contour on boundaries. Contour intervals $=0.05$. (Dimension of plate not to scale) 


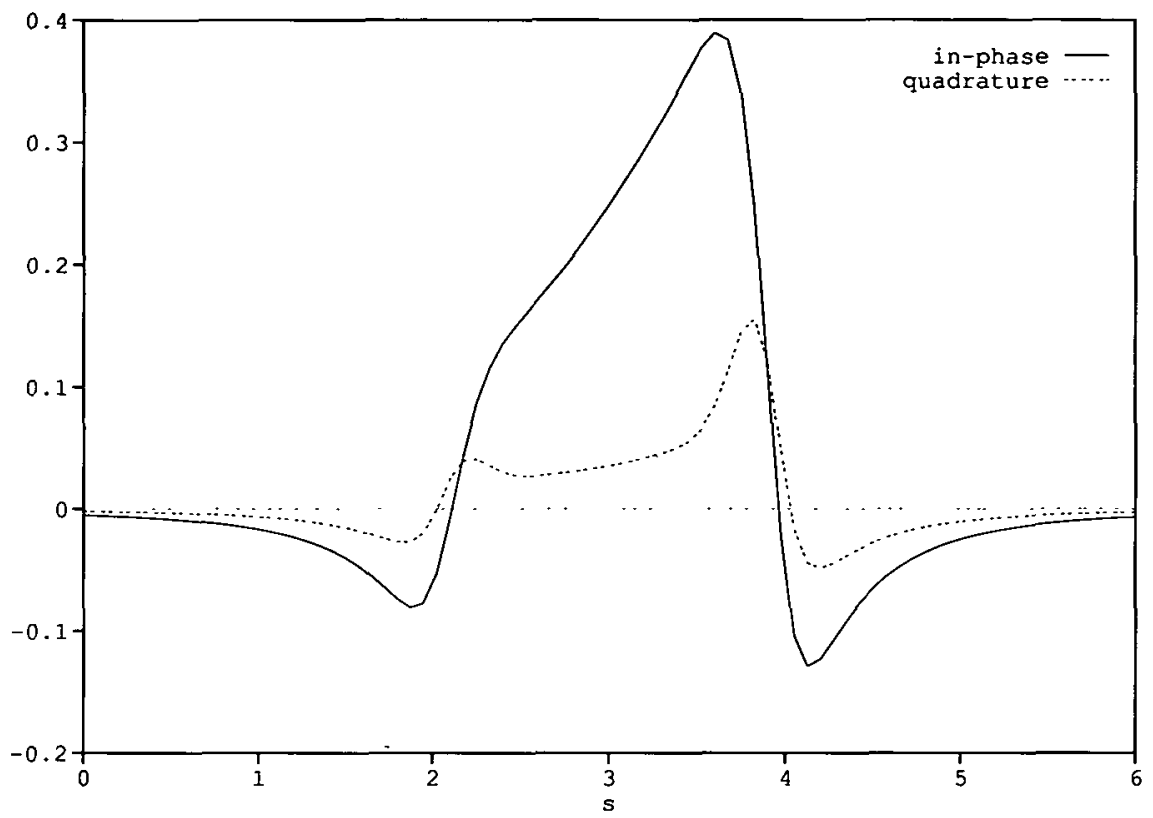

FIGURE 4. Vertical component of the induced magnetic field for the rectangular plate due to a unit dipole at $(1.5,4,0.2) . \alpha=20$. The horizontal scale measures distances from $(1.5,-2,0.2)$ to $(1.5,4,0.2)$. The plate occupies the same region as in Figure 2.

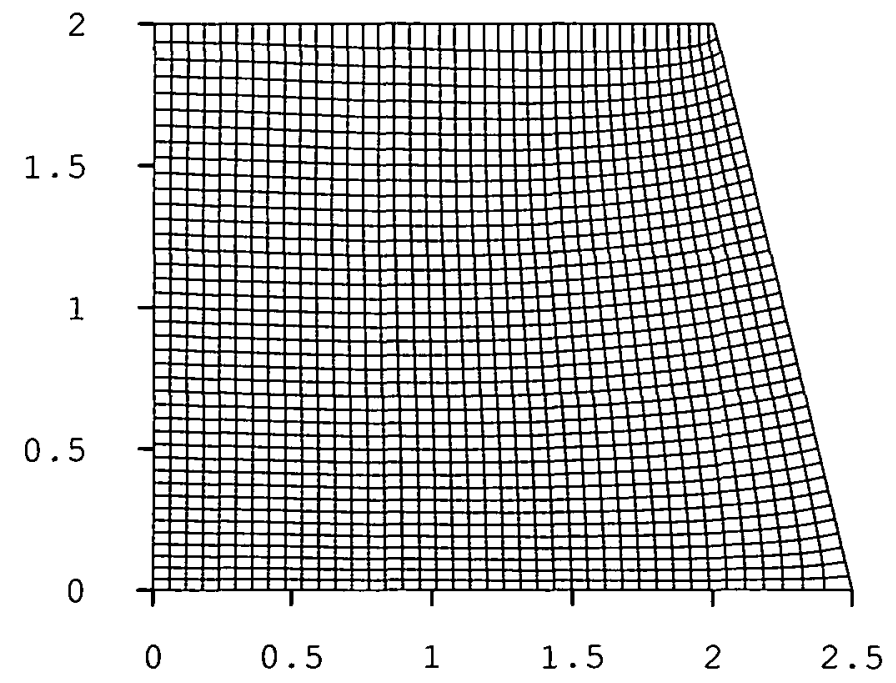

FIGURE 5. $40 \times 40$ grids for a quadrilateral $\Delta f=0.00928, M D O=0.0895$. 


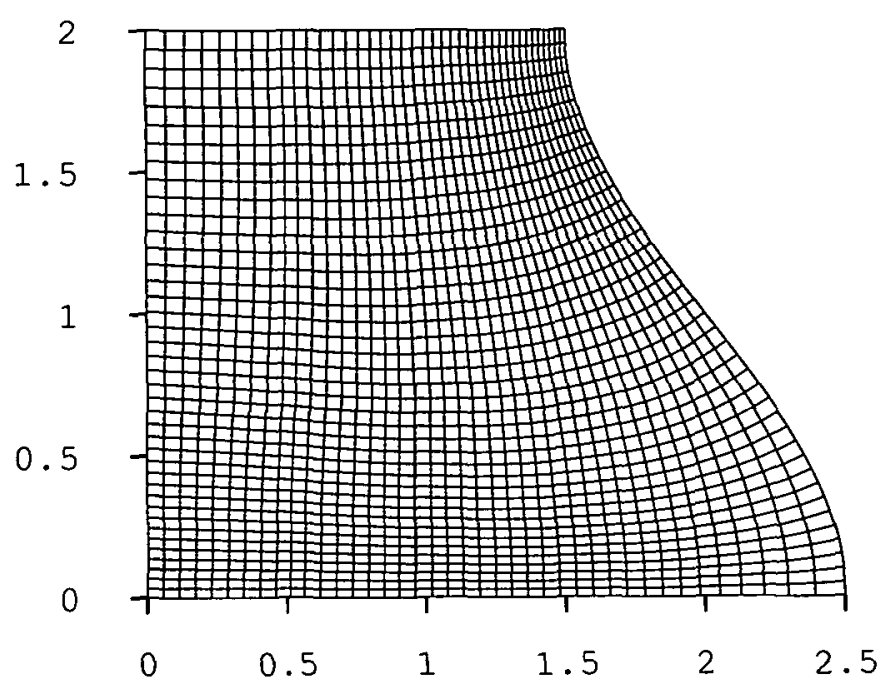

FIGURE 6. $40 \times 40$ grids for an otherwise rectangular shape. Right hand boundary has equation $x=2+0.5 \cos (\pi y / 2) . \Delta f=0.00994, M D O=0.0155$.
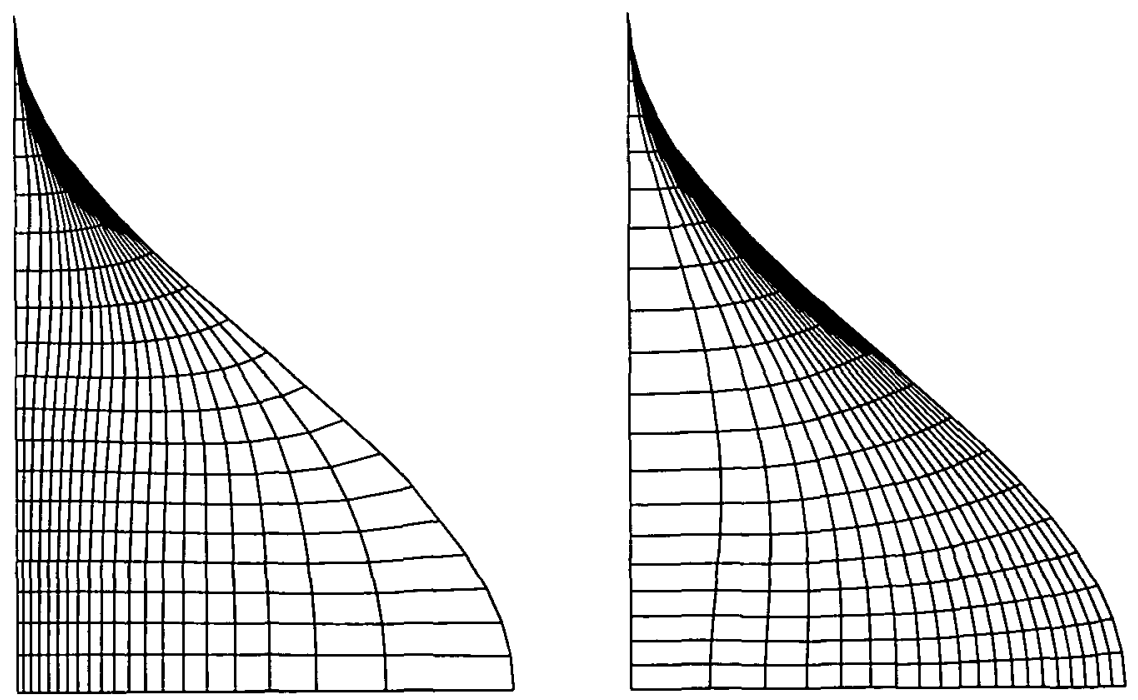

FIGURE 7. $20 \times 20$ grids generated using two different distortion functions. On the left $f$ is defined by (4.4). On the right $f$ is given by (4.4) multiplied by $(1-0.2 \cos \pi \eta)$. 


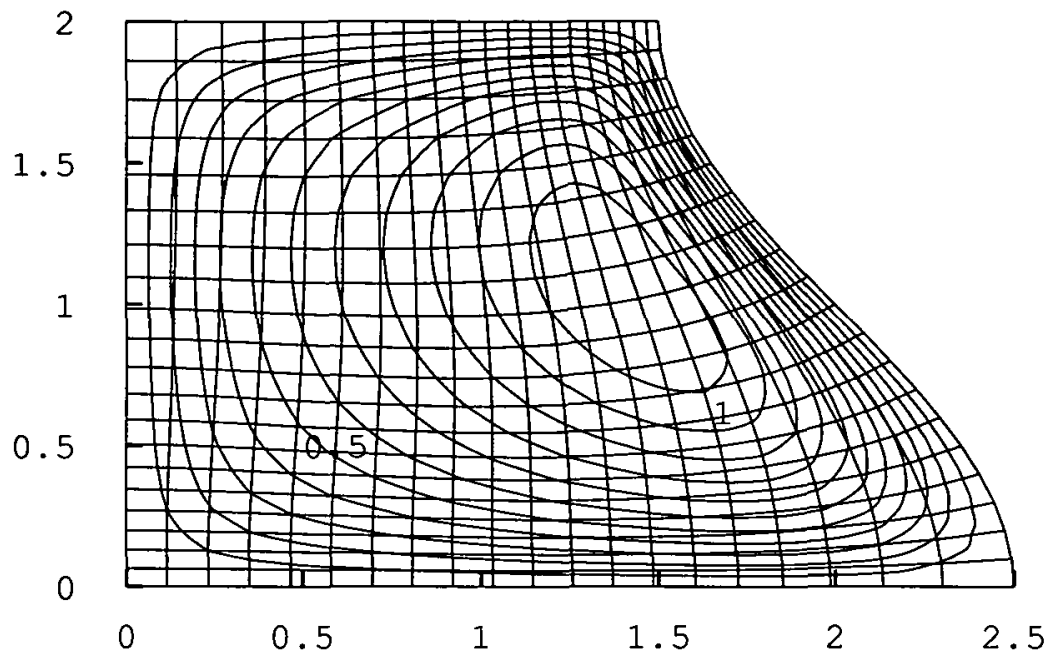

(a)

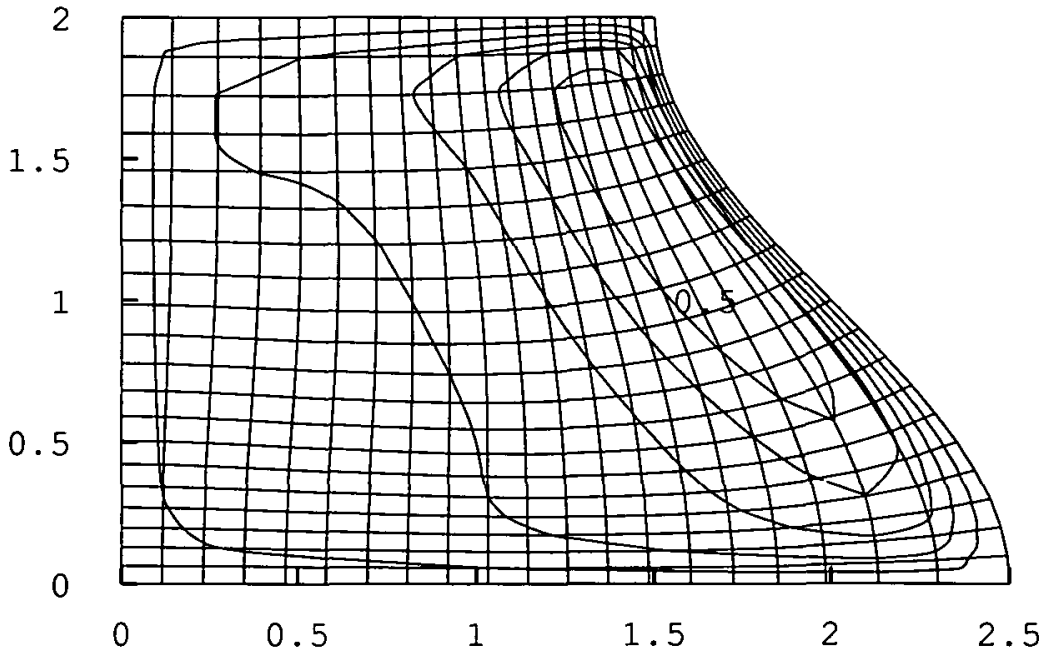

(b)

FIGURE 8. Stream potential maps for the region of figure 6 with a unit vertical dipole situated at $(3,1,0.2)$. (a) In-phase component. (b) Quadrature component. $\alpha=20$. Zero contour on boundaries. Contour intervals $=0.1$. 


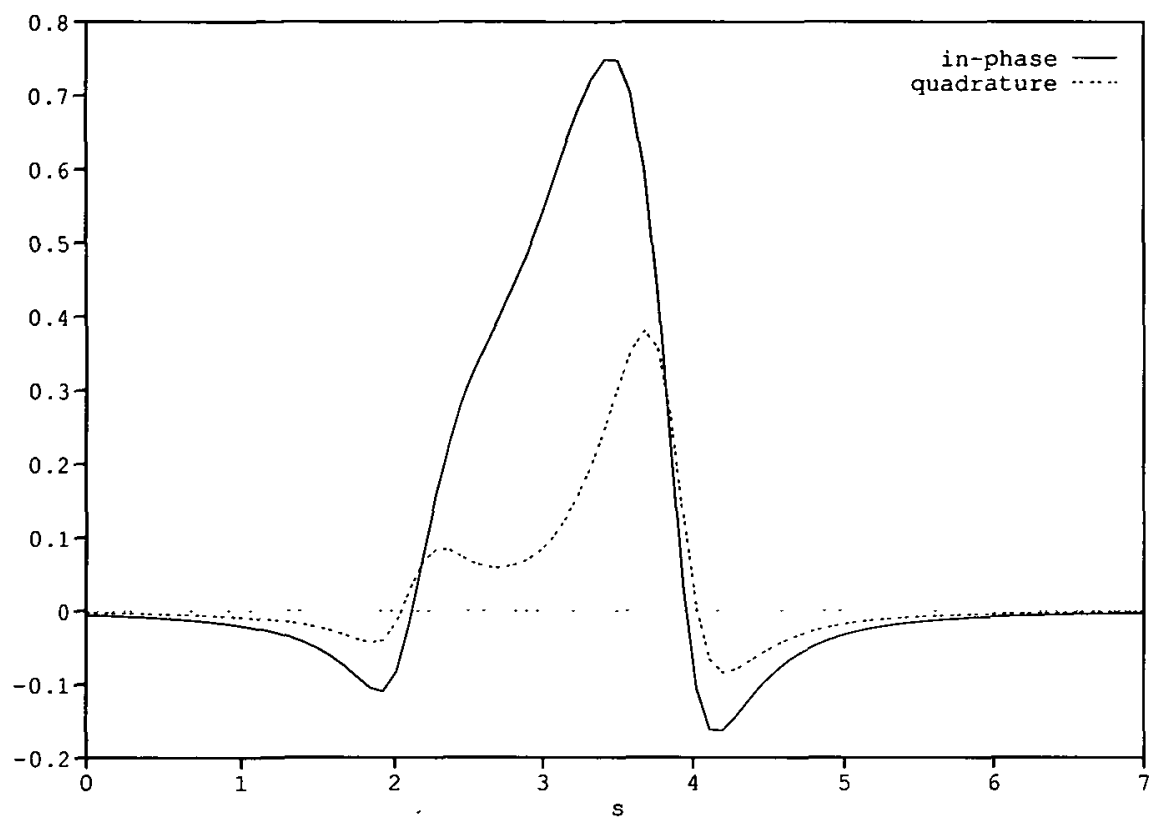

FIGURE 9. Vertical component of the induced magnetic field. The inducing dipole is at $(3,1,0.2), \alpha=20$. The horizontal scale $s$ measures distances along the path of traverse from $(-2,1,0.2)$ to $(5,1,0.2)$.

\section{Discussion}

We have treated the problem of a thin planar conductor in free space. The method is relevant for deposits whose lateral extent is much larger than its thickness. It should be noted that in practical situations we need to take account of the surrounding medium unless of course the host turns out to be only weakly conducting. When the host medium is also conducting, the problem is best tackled by reformulating in terms of the electric field, resulting in an integral equation (see [13]) which may be treated in a manner similar to that described here. We note from the magnetic field response that the curves typically peaks near the edge of the conductor, not unlike the type curves associated with magnetic surveys. This property may be used to ascertain the lateral extent of the deposit by taking sufficient numbers of readings from various lines of traverse. The present formulation does not suggest a ready method for depth estimation.

The advantage of the method presented in this paper lies in its simplicity. 
Every region is mapped onto a unit square and the corresponding problem solved in the transformed plane over a regular mesh. The grid generated can be varied by imposing different expressions for the distortion function as well as by changing the choice of boundaries on which to impose Neumann or Dirichlet conditions. The accuracy obtainable is affected by two factors. One is the accuracy with which the orthogonal grids can be generated numerically. For shapes that do not have corners that differ too much from the right angle, one can expect to get good orthogonal grids. This is inherent in the method used here. Another factor is the interpolation quadrature for $U$. To keep the algebra simple, we have used a quadrature that is linear in the two coordinates. The cancellation in $I_{4}$ for each interior point occurs over a regular mesh in the transformed plane irrespective of its actual location in the physical plane.

\section{Acknowledgement}

This work has been partially supported by a Curtin Research Grant. The author wishes to acknowledge the contribution of Mr Peter Staples who helped to implement the numerical codes used in this paper.

\section{Appendix}

In this appendix, we will justify neglecting the contribution $I_{4}$, from the four squares $\Delta$ say, surrounding the observation point $\left(x_{m n}, y_{m n}\right)$, where $x_{m n}=$ $x\left(\xi_{m}, \eta_{n}\right)$ and $y_{m n}=y\left(\xi_{m}, \eta_{n}\right)$. Starting with (5.1a) the integral over $\Delta$ is given by

$$
\begin{aligned}
I_{4}=\int_{(n-1) h}^{(n+1) h} \int_{(m-1) h}^{(m+1) h}\{[ & \left.\left(x_{m n}-x^{\prime}\right) \frac{\partial y^{\prime}}{\partial \eta^{\prime}}-\left(y_{m n}-y^{\prime}\right) \frac{\partial x^{\prime}}{\partial \eta^{\prime}}\right] \frac{\partial U}{\partial \xi^{\prime}} \\
& \left.-\left[\left(x_{m n}-x^{\prime}\right) \frac{\partial y^{\prime}}{\partial \xi^{\prime}}-\left(y_{m n}-y^{\prime}\right) \frac{\partial x^{\prime}}{\partial \xi^{\prime}}\right] \frac{\partial U}{\partial \eta^{\prime}}\right\} \\
& \times\left[\left(x_{m n}-x^{\prime}\right)^{2}+\left(y_{m n}-y^{\prime}\right)^{2}\right]^{-3 / 2} d \xi^{\prime} d \eta^{\prime},
\end{aligned}
$$

where $x^{\prime}, y^{\prime}$ are functions of $\xi^{\prime}$ and $\eta^{\prime}$ and $h$ is the mesh size as before. When the conductor is a square, then $\partial x^{\prime} / \partial \eta^{\prime}=0=\partial y^{\prime} / \partial \xi^{\prime}$ and the integrand in (A.1) simplifies somewhat. When the mesh size is small enough, we expect that both $x^{\prime}$ and $y^{\prime}$ may be approximated closely by linear functions of $\xi^{\prime}$ and $\eta^{\prime}$ 
in the region $\Delta$ irrespective of the shape of the conductor. This means higher derivatives of $x^{\prime}$ and $y^{\prime}$ may be neglected and hence the distortion function $f$ may be assumed constant throughout this region. This linear relation is in fact exact when the conductor is rectangular in shape. For conductors of large extent this fact may be exploited by dividing the region into subregions which contain rectangles. We now let

$$
\xi^{\prime}=m h+\bar{\xi} h, \quad \eta^{\prime}=n h+\bar{\eta} h,
$$

and then, over $\Delta$ we have

$$
x^{\prime}\left(\xi^{\prime}, \eta^{\prime}\right)=x_{m n}+\bar{\xi} h \frac{\partial x^{\prime}}{\partial \xi^{\prime}}+\bar{\eta} h \frac{\partial x^{\prime}}{\partial \eta^{\prime}}
$$

so that

$$
x_{m n}-x^{\prime}=-h\left[\bar{\xi} \frac{\partial x^{\prime}}{\partial \xi^{\prime}}+\bar{\eta} \frac{\partial x^{\prime}}{\partial \eta^{\prime}}\right]
$$

Similarly

$$
y_{m n}-y^{\prime}=-h\left[\bar{\xi} \frac{\partial y^{\prime}}{\partial \xi^{\prime}}+\bar{\eta} \frac{\partial y^{\prime}}{\partial \eta^{\prime}}\right] .
$$

Substituting into (A.1) now yields

$$
I_{4}=-\int_{-1}^{1} \int_{-1}^{1} \frac{\left[\bar{\xi} \frac{\partial U}{\partial \bar{\xi}}+\bar{\eta} \frac{\partial U}{\partial \bar{\eta}}\right] f}{h\left[\bar{\xi}^{2}+\bar{\eta}^{2} f^{2}\right]^{3 / 2}} d \bar{\xi} d \bar{\eta}
$$

where (4.5) and the relation $J=f h_{1}^{2}$ from Section 5 have been used. Using a bilinear quadrature for $U$ based on the corner points of $\Delta, v i z$.

$$
\begin{aligned}
U\left(\xi^{\prime}, \eta^{\prime}\right)= & \left\{\left[\xi^{\prime}-(m-1) h\right]\left[\eta^{\prime}-(n-1) h\right] U_{m+1, n+1}\right. \\
& -\left[\xi^{\prime}-(m-1) h\right]\left[\eta^{\prime}-(n+1) h\right] U_{m+1, n-1} \\
& +\left[\xi^{\prime}-(m+1) h\right]\left[\eta^{\prime}-(n+1) h\right] U_{m-1, n-1} \\
& \left.-\left[\xi^{\prime}-(m+1) h\right]\left[\eta^{\prime}-(n-1) h\right] U_{m-1, n+1}\right\} /\left(4 h^{2}\right),
\end{aligned}
$$

we see that (A.5) is made up of three principal value integrals with integrands which are proportional to $\bar{\xi}\left[\bar{\xi}^{2}+\bar{\eta}^{2}\right]^{-3 / 2}, \bar{\eta}\left[\bar{\xi}^{2}+\bar{\eta}^{2}\right]^{-3 / 2}$ and $\bar{\xi} \bar{\eta}\left[\bar{\xi}^{2}+\bar{\eta}^{2}\right]^{-3 / 2}$. Each improper integral converges to zero. 


\section{References}

[1] A. A. Ashour, "Electromagnetic induction in thin finite sheets having conductivity decreasing to zero at the edge, with geomagnetic applications I", Geophys. J. R. Astr. Soc. 22 (1971) 417-443.

[2] E. D. Chikhliwala and Y. C. Yortsos, "Application of orthogonal mapping to some twodimensional domains", J. Comput. Physics 57 (1985) 391-402.

[3] B. Fornberg, "A numerical method for conformal mappings", SIAM J. Sci. Stat. Comput. 1 (1980) 386-400.

[4] D. G. Hurley and P. F. Siew, "The decay of eddy-currents in thin sheets and related waterwave problems", IMA J. Appl. Math. 34 (1985) 1-21.

[5] I. S. Kang and L. G. Leal, "Orthogonal grid generation in a 2D domain via the boundary integral technique", J. Comp. Phys. 102 (1992) 78-87.

[6] Y. Lamontagne and G. F. West, "EM response of a rectangular thin plate", Geophysics 36 (1971) 1204-1222.

[7] A. T. Price, "The induction of electric currents in non-uniform thin sheets and shells", Qtr J. Mech. Appl. Math. 2 (1949) 282-310.

[8] G. Ryskin and L. G. Leal, "Orthogonal mapping", J. Comput. Physics 50 (1983) 71- 100.

[9] P. F. Siew, "Second order effects in the induction of thin discs", IMA J. Appl. Math. 48 (1992) 97-106.

[10] P. F. Siew and D. G. Hurley, "Electromagnetic response of thin discs", J. Appl Math. Physics (ZAMP) 39 (1988) 619-633.

[11] W. R. Smythe, Static and dynamic electricity (McGraw-Hill, London, 1968).

[12] A. H. Stroud and D. Secrest, Gaussian quadrature formulas (Prentice-Hall Inc, 1966).

[13] G. F. West and R. N. Edwards, "A simple parametric model for the electromagnetic response of an anomalous body in a host medium", Geophysics 50 (1985) 2542-2557. 